\title{
The precision cutting control research of automotive stainless steel thin wall pipe
}

\author{
Lihong Jin ${ }^{1, a}$, Shaotai Deng ${ }^{2}$, Chuxiong Xie ${ }^{2}$, Zhishen Wang ${ }^{2}$, Chunfu GAO ${ }^{2}$ \\ ${ }^{1}$ Zhejiang Wanliyang Transmission Co., Ltd.Dongyang, Zhejiang Prov, China \\ ${ }^{2}$ College of Engineering, ZheJiang Normal University, 321004, Jinhua, China
}

\begin{abstract}
Stainless steel thin-walled tube are widely used in automobile industry at present, but as a result of thin wall pipe is poor strength and poor rigidity, which lead to deformation, shaped differencer and other problems in the process, it is hard to ensure the processing quality of parts. This paper proposes a method of thin stainless steel thin wall pipe cutting process in vehicle, greatly improved the problems and technical difficulties in the traditional process, the main research is about the cutting system and the hydraulic fixture design, obtained under low cost circumstances, it can realize high precision stainless steel pipes, high degree of automation to automatic cutting,simplified operation steps at the same time, increased the applicability of the system, provided a kind of advanced stainless steel thin wall pipe cutting device for the small and medium-sized enterprises.
\end{abstract}

\section{Introduction}

stainless steel materials because of the high strength, relatively lighter quality, especially after the impact, energy absorption is better than other metal materials, the performance of the safety is good, and compared with the ordinary carbon steel vehicles, which has the advabtage in light weight, long service life, simple manufacturing assembly process etc, the stainless steel with the development of automobile industry,it applys in the automotive industry, which is more and more widely, in the frame, exhaust systems, engine parts etc for the importance of the increasingly prominent. But cutting stainless steel thin wall tube makes mold in the process field, trimming and punching is the existence of technical bottlenecks, particularly some complex structure of Large car mold oblique cutting, oblique shock for the big car mold. The determination of the trimming line requires repeated dozens of times to complete, which brought great efforts to fitters and cutting machine, Not only put forward higher requirements for the skill level of the operator's fitters, but also seriously affect the after process of the mold to debug.

\section{Designing stainless steel thin-walled pipe cutting machine system design}

\subsection{The systematic mechanical structure design of the stainless steel thin wall pipe cutting machine}

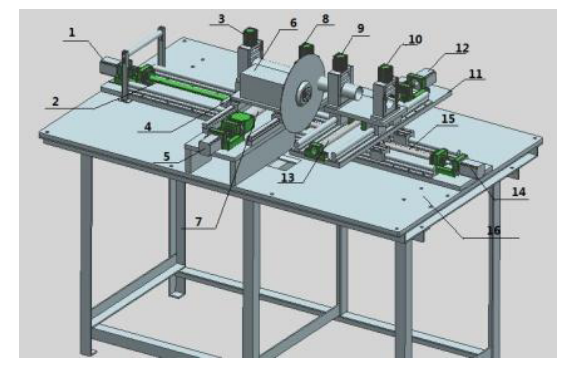

1 Feed drive motor 2 Feed drive screw 3 Feed fixture 4 Cutting table 5 Cutting feed motor 6 Cutting motor 7 Cutting feed screw 8 Cutting fixture 9 Deburring stage fixtures 10 Deburring after fixture 11 Deburring station 12 Deburring $\mathrm{X}$-axis drive motor 13 Deburring $\mathrm{X}$-axis drive screw 14 Deburring Y-axis drive motor 15 Deburring Y-axis drive screw 16 Deburring device

Figure 1. Systematic mechanical structure

Stainless steel thin wall pipe cutting machine is horizontal structure, mechanical equipment is mainly composed of the mechanical body, transmission device,

$\overline{{ }^{a} \text { Lihong Jin: 757987@163.com }}$ 
cutting device and deburring device of the four major part. See systematic mechanical structure as shown in figure 1.

Automotive stainless steel thin wall pipe cutting precision cutting device works principle as follows: stayed on the left of the limit position of the feeding fixture to operate, clamping stainless steel thin-wall workpiece, feed drive screw is whirled by feed drive motor through the coupling, feed drive screw is connected with the base fixture of the installed semicircle guideway through screw nut, so it can realize precise linear feed; When the feed drive rod reaches the right limit position, cutting fixture, deburring stage fixture and deburring after fixture clamps the workpiece of stainless steel thin-wall, geared motors is fixed on cutting table to operate at the same time, according to the thickness of the workpiece, the operator can realize deceleration motor speed regulation through the machine's own debugger; Cutting drive motor drives cutting feed screw to whirl through the coupling, cutting feed screw is connected with the base fixture of the installed semicircle guideway through the screw nut, the movement of cutting machine can realize wheel tablets to cut feed and stably linear feed, which can avoid cutting swinging from cutting damaging; When cutting has been completed, the cutting feed motor is reversed to realize the replacement of cutting machine; The $\mathrm{X}$ axis motor of deburring machine makes the $\mathrm{X}$ axis screw of deburring machine whirl through coupling, the $\mathrm{X}$ axis screw of the deburring machine is connected with the base fixture of the installed semicircle guideway through the screw nut, realizing the left lateral movement of the deburring table, achieving stainless steel thin wall pipes to reach the longitudinal central; The $\mathrm{Y}$ axis motor of deburring machine drives the $\mathrm{X}$ axis screw of deburring machine to whirl, screw is connected with the installed semicircle rails with the supporting base of the installed deburring workbench through screw nut, realizing the deburring workbench to move forward, achieving workpiece to reach the centering feed or accurately, realizing the deburring process of the front cutting,finishing the deburring process of the front cutting,controling the motor of the deburring $\mathrm{X}$ axis and the deburring $\mathrm{Y}$ axis to reverse and realize the deburring process of the after incision; The system realizes the automatic completion of the whole process,it's high stability, high precision and good safety performance.

\subsection{Designing the control system of cutting machine}

Intelligent control system ${ }^{[4]}$ solves the problem of complex control system through the integration of hardware and software,but traditional control system couldn't solve; Hardware is the basis of the control system in system, software is the soul of the control system, system operates well under the action of the software to complete the functional requirements of the system. The parts of hardware often include controller, sensors, actuators, power supply, channels of Input and output 1 , etc.

Automatic control system is the core part of stainless steel thin wall pipe cutting machine, the design of the control system directly determines the efficiency and precision of the cutting machine, the system is divided into the PLC control system module, motor drive module, hydraulic fixture control module, position sensor module.

According to the requirements of the electrical control system,electrical part of the overall scheme of stainless steel thin-walled pipe cutter shown in Figure 2, the PLC control system is chosen as the core control system, microcontroller system is used for the control system of the electric auxiliary system.

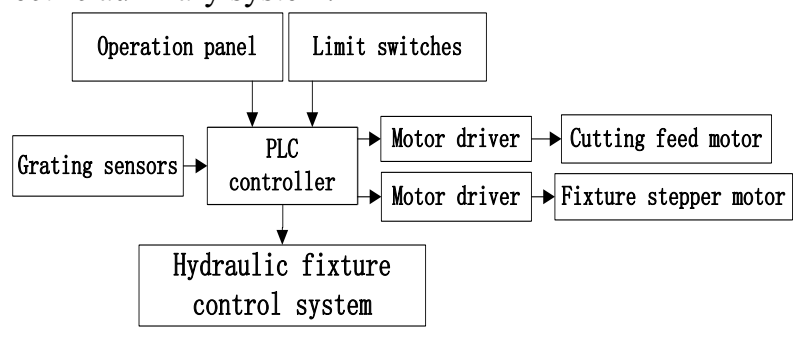

Figure 2. Control system structure drawing

As can be seen from Figure 2, various switch control information and detection signal is input to the PLC in the main control system, the information calculated and judged by the PLC program, then the information is output to the actuator by PLC, realizing the motion control of thin-walled stainless steel tube cutting .PLC selects DVP-10 sx in figure 2 , it only has 10 external ports in DVP-10SX, which can not meet the port requirements of the control system,so it needs extension modules to increase the external ports.DVP-16SP11T has input and output modules, which has 8 input and 8 output expansion modules, the output type is transistor, DVP16SP11T extension modules can be connected directly with the host of the DVP-10SX, it has small size and installs easily. Cutter feed drives select the stepper motor of 86 series, this type is $86 \mathrm{BYGH} 9820$. Stepper motor has three basic drive modes:the whole step, half-step, segmentation model.System needs to achieve precise location, So selecting segmentation model to drive stepping motor; fixture drive stepper motor drive selects two-phase hybrid stepping motor driver DM542A of subdivision type.According to the characteristics of the cutting feed drive motor,choosing DM860A that is twophase hybrid drives of segment type.The raster position sensor selects model for the JCXF1, JCXF1 adopts advanced grating measurement technology, which has excellent repeated positioning and a high level of measurement accuracy.

\section{The design of hydraulic fixture}

\subsection{Fixture selection}

Stainless steel thin-walled tubes will be subject to the cutting grinding wheel of the main cutting force and radial feed force in cutting process, which needs to guarantee verticality, otherwise it will affect cutting accuracy, the cutting the wheel tablets will be damaged at the same time, so the design of fixture is very critical part of the cutter design, the performance stainless steel thinwalled tube cutter largely depends on the design of the 
fixture. According to the requirements of the system, This article fixture design shown in Figure 4.

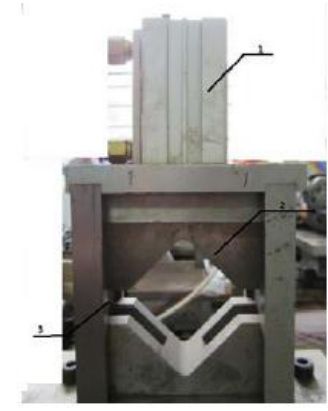

1 , hydraulic cylinder 2 , fixture body 3 , guide-track groove

Figure 3 . Fixture structure

The fixture is composed of hydraulic cylinder, fixture body, guide-track groove, fixture body is embedded in the guide-track groove, which is connected with hydraulic cylinder,the hydraulic clamp device to control the action; This fixture is installed symmetrically on both sides of the cutting wheel, when cutting stainless steel,it can guarantee the force equilibrium of the sample, which prevents the sample truncating, avoiding a situation tear to affect the cutting neat; In order to meet the system design,the fixture has the following advantages:(1)the fixture structure is simple to manufacture easily, the cost is low;(2)installed easily;(3)it can satisfy different types clamping of stainless steel thin wall pipe .

\subsection{The design of fixture hydraulic control system}

Combining with the requirements of fixture system process characteristics in this paper,the electro-hydraulic control technology is brought into the control system,system composition diagram shown in Figure 4.Electro-hydraulic control system of Walled tube fixtures includes a microcontroller minimum system, position sensors, pressure sensors, fixtures systems, hydraulic output system, the proportional solenoid valve.Pressure sensors and position sensors gathers the information of the fixtures of location and pressure in real-time, because the pressure information is too small, which is treated through the amplification circuit, signal enters into the $A / D$ converter port to switch after conditioning, and then it enters into the smallest Microcontroller system, the microcontroller treats the sample data,it is delivered to the LCD to display, according to the position information and pressure information,microcontroller controls proportional solenoid valve, which decides it to turn on or turn off,so as to realize the control of the fixture system, single chip microcomputer system communicates with PC through RS232.

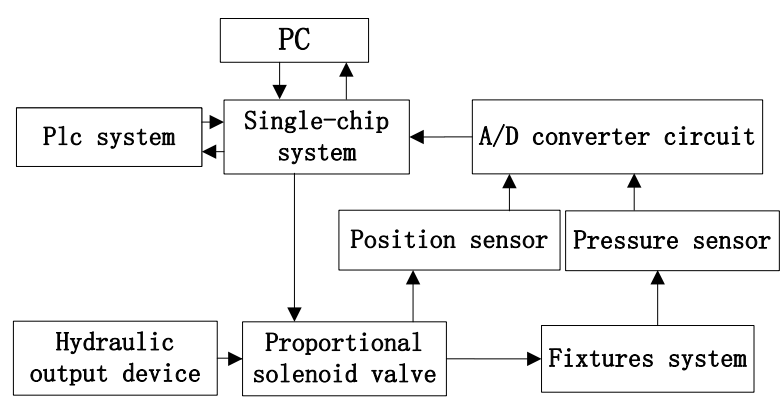

Figure 4. Hydraulic fixture control system schematic diagram

\section{The choice of cutting tools and cutting way}

The commonly used methods of plant have plasma arc cutting methods, laser cutting, generating method, progressive ring knife cutting method ,blade cutter cutting for thin-walled tube cutting current.The plasma arc cutting requires a high temperature to realize a metal fuse, which will cause incision melted.Incision of the laser cutting is shaped well and narrow kerf,but the cost is high.Generating method and circle knife gradual cutting method is used to cut,since the incision subject to greater pressure, the incision shows a certain degree of deformation, mechanical structure is too complicated at the same time, it does not comply with simple principles of mechanical design.

This paper selects cutter blade cutting method, but stainless steel blade cutting process appears steel strong adhesion of the main problems in the processing engineering,chips easily attached to the blade serrated,making the cutting conditions deteriorate, which accelerates the speed of the blade bluntness. According to the requirements of the system cutting function, it chooses HSS-M42 models, which has high speed steel cutting special hacksaw of containing cobalt,the size is $275^{*} 1.6 * 32 \mathrm{~mm}$. This blade is coated with a surface coating of chromium nitride alloys, which makes the performance the blade wear-resistance to get stronger,it reduces the high speed movement of resistance at the same time, which can prolong the service life of saw blade and maintain long-term stability, it effectively improves the stability of saw blade, hardness and wear-resistance.

\section{Experiment}

Experiment verifies that plasma arc heat realizes the method of metal fuse, because excessive temperature leads to thin wall pipe incision melt,Fusing effect shown in Figure 5. While using the cutting method of saw blade cutter,deviceruns continuously, debugging program, debugging the feed speed and cutting speed,making the system reach the optimum under the current conditions. 


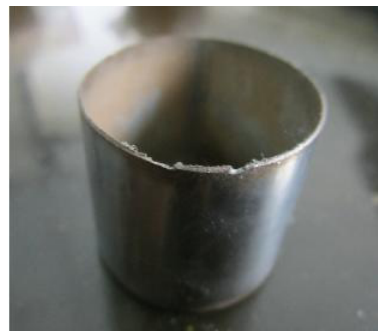

Figure 5. Plasma incision

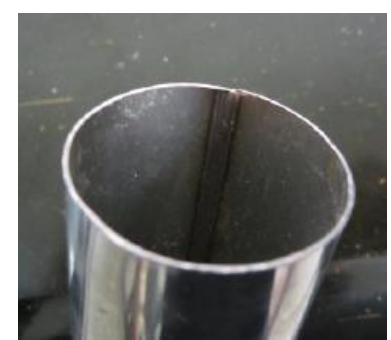

Figure 6. The saw blade cutter
The cutting method of saw blade cutter can better meet the requirements, and incision formation,don't lead to deformation, it has not much burr after cutting. The cutting effect is shown in Figure 6 after cutting, automotive stainless steel thin-walled tube precision cutting device has simple structure, the low cost, high precision, high degree of automation, this device is affordable, cost-effective for small and medium-sized enterprises, which can improve production efficiency, bring more economic benefits for the enterprise.

\section{Conclusion}

According to the requirements, installing and debugging the whole system, the whole machine performance test, hydraulic fixture test and security measure test; it can achieve the design of functional requirements by debugging the system, while performance tests to verify the performance of thin-walled stainless steel tube cutting machine,hydraulic fixtures control performance and safety measures can better meet the requirements of the design.Stainless steel thin-walled pipe precision cutting device has high automation, the low cost, high precision, reliable characteristics, it will be widely used in automotive stainless steel thin wall pipe cutting In the future development trendency.

\section{References}

1. $\mathrm{Xu}$ Qingjing.Developers portable CNC cutting machine control system.D.(2007)

2. $\mathrm{Xu}$ Yan. large diameter cylinder $\mathrm{CNC}$ pipe cutting equipment research.D.(2008)

3. Huang Jian,Wo Ding,Yi et.CNC pipe cutting machine developed mechanical industrial automation . J .15,2 (1993).

4. Luo xueke, Xie Fuchun. NC principle and CNC machine tools .Beijing: Chemical Industry Press Industrial Equipment and Information Engineering Publishing Center.M.( 2004)

5. Xie Chuxiong .stainless steel thin-walled tube cutting system.D.(2014)

6. Guya Lei. Automatic precision cutting machine and the study of control system.D.( 2004)

7. Yan liangjun, $\mathrm{Li}$ Guoping. The research and development of thinly walled tube fast automatic cutting machine.J.Machine Building \& Automation. 34 .2(2007)

8. Yang Suyuan,Pan wenfang. The research and application of $\mathrm{CNC}$ cutting technology .J. metal processing: Thermal processing.37 .8(2010)
9. Li Weiqi.The improvement of the pipeline cutting technology .J.installation.37. 2 (2005)

10. Ha Lei. The pipe cutting system design based on PLC .D. (2012)

11. Wang Zhengchao.The design of cutting machine motion control system based on PLC .D. Zhengzhou University .(2011)

12. Yang Jian.The system complex control of electrohydraulic proportional position and related research .D. Hangzhou: Zhejiang University.( 2005)

13. Liu Zhong, Long Guojian.The pressure control system design of based on the high-speed switch electromagnetic valve technology .J. Journal of hydraulic and pneumatic.13. 2(2003) 